\title{
Association of Newly Developed Right Bundle Branch Block with Graft Rejection Following Heart Transplantation
}

\author{
Jin-Ho Kim ${ }^{1,2 *}$, Jaewon $\mathrm{Oh}^{1 *}$, Min Ji Kim ${ }^{1}$, In-Cheol Kim³ ${ }^{3}$ Jae-Sun Uhm ${ }^{1}$, Hui-Nam Pak ${ }^{1}$, and Seok-Min Kang ${ }^{1}$ \\ ${ }^{1}$ Division of Cardiology, Severance Cardiovascular Hospital, Cardiovascular Research Institute, Yonsei University College of Medicine, Seoul; \\ ${ }^{2}$ Departement of Cardiology, Konkuk University School of Medicine, Chungju; \\ ${ }^{3}$ Departement of Cardiology, Keimyung University School of Medicine, Daegu, Korea.
}

Purpose: We aimed to examine associations between right bundle branch block (RBBB) following heart transplantation (HT) and graft rejection.

Materials and Methods: We investigated 51 patients who underwent endomyocardial biopsies, electrocardiogram, right-side cardiac catheterization, and echocardiography at 1 month and 1 year after HT. We classified patients into four groups according to the development of RBBB, based on electrocardiogram at 1 month and 1 year: 1) sustained RBBB, 2) disappeared RBBB, 3) newly developed RBBB, and 4) sustained non-RBBB. The RBBB was defined as an RSR' pattern in V1 with a QRS duration $\geq 100 \mathrm{~ms}$ on electrocardiogram.

Results: The newly developed RBBB group ( $\mathrm{n}=13,25.5 \%)$ had a higher rate of new onset graft rejection (from grade 0 to grade $\geq 1 \mathrm{R}$, $30.8 \%$ vs. $10.0 \%$ vs. $21.4 \%, p=0.042)$ at 1 year, compared with sustained $\mathrm{RBBB}(\mathrm{n}=10,19.6 \%)$ and sustained non-RBBB group ( $=28$, $54.9 \%$ ). In contrast, the incidence of resolved graft rejection (from grade $\geq 1 R$ to grade 0 ) was higher in the sustained RBBB group than the newly developed RBBB and sustained non-RBBB groups ( $70.0 \%$ vs. $7.7 \%$ vs. $25.0 \%, p=0.042)$. Left atrial volume index was significantly higher in the newly developed RBBB group than the sustained RBBB and sustained non-RBBB groups $\left(60.6 \pm 25.9 \mathrm{~mL} / \mathrm{m}^{2}\right.$ vs. $36.0 \pm 11.0 \mathrm{~mL} / \mathrm{m}^{2}$ vs. $38.4 \pm 18.1 \mathrm{~mL} / \mathrm{m}^{2}, p=0.003$ ).

Conclusion: Close monitoring for new development of RBBB at 1 year after HT, which was associated with a higher incidence of new onset graft rejection, may be helpful to identify high risk patients for graft rejection.

Key Words: Right bundle branch block, heart transplantation, rejection

\section{INTRODUCTION}

A right bundle branch block (RBBB) is a common electrocardiographic abnormality in patients with heart transplantation

Received: November 6, 2018 Revised: February 16, 2019

Accepted: March 13, 2019

Corresponding author: Seok-Min Kang, MD, PhD, Division of Cardiology, Severance Cardiovascular Hospital, Yonsei University College of Medicine, 50-1 Yonsei-ro, Seodaemun-gu, Seoul 03722, Korea.

Tel: 82-2-2228-8450, Fax: 82-2-2227-7732, E-mail: smkang@yuhs.ac

* Jin-Ho Kim and Jaewon Oh contributed equally to this work.

- The authors have no potential conflicts of interest to disclose.

(c) Copyright: Yonsei University College of Medicine 2019

This is an Open Access article distributed under the terms of the Creative Commons Attribution Non-Commercial License (https://creativecommons.org/licenses/ by-nc/4.0) which permits unrestricted non-commercial use, distribution, and reproduction in any medium, provided the original work is properly cited.
(HT), with a prevalence rate of $12-79 \% .^{1-4}$ Although limited studies have reported conflicting clinical outcomes, the occurrence of RBBB after HT appears to be associated with poor clinical outcomes, including increased mortality, especially in patients with progressive RBBB. ${ }^{4-6}$ Small studies have described inflammatory infiltration related with graft rejection in the conduction system, as well as in the myocardium. Foerster ${ }^{7}$ reported that the conduction system was involved in 8 cases among 11 allografts with acute graft rejection, and Calzolari, et al. ${ }^{8}$ reported that complete or incomplete RBBB was founded in 11 cases out of 12 transplanted hearts with acute or chronic rejection in necropsy histologic study. Chan, et al. ${ }^{9}$ indicated that conduction abnormalities including RBBB could be associated with rejection, which might result in worse clinical outcomes. 
However, there were not enough data about the patterns of development of RBBB and its relation with graft rejection and hemodynamic parameters following HT. Therefore, we sought to investigate the time-course development of RBBB on electrocardiogram (ECG) and its association with graft rejection, echocardiographic parameters, and invasive hemodynamic parameters in HT patients.

\section{MATERIALS AND METHODS}

\section{Study population}

We performed a retrospective analysis of 79 patients who underwent bicaval orthotopic HT at Severance Cardiovascular Hospital from 2011 to 2015. The study protocol was approved by the Institutional Review Board of Severance Hospital (No. 4-2013-0665). All patients received basiliximab induction therapy, followed by triple immunosuppressive therapy (tacrolimus, mycophenolate mofetil, prednisolone). We excluded patients $(\mathrm{n}=17)$ who died due to fatal infection or multi-organ failure within 1 month and patients $(n=11)$ who did not undergo either endomyocardial biopsy, echocardiography, or right-side cardiac catheterization at 1 month and 1 year after HT. Finally, we analyzed 51 patients who underwent all of previously mentioned exams at 1 month and 1 year after HT (Fig. 1).

Based on ECG at 1 month and 1 year following HT, four groups of patients were identified: 1) sustained RBBB group (RBBB at 1 month and remaining RBBB at 1 year), 2) disappeared RBBB group (RBBB at 1 month and ECG at 1 year showed nonRBBB), 3) newly developed RBBB group (ECG at 1 month was non-RBBB, but ECG at 1 year showed RBBB), and 4) sustained non-RBBB group (ECG at 1 month was non-RBBB and remaining non-RBBB at 1 year).

\section{Electrocardiography and endomyocardial biopsy}

Standard 12-lead ECGs were obtained in the hospital after HT

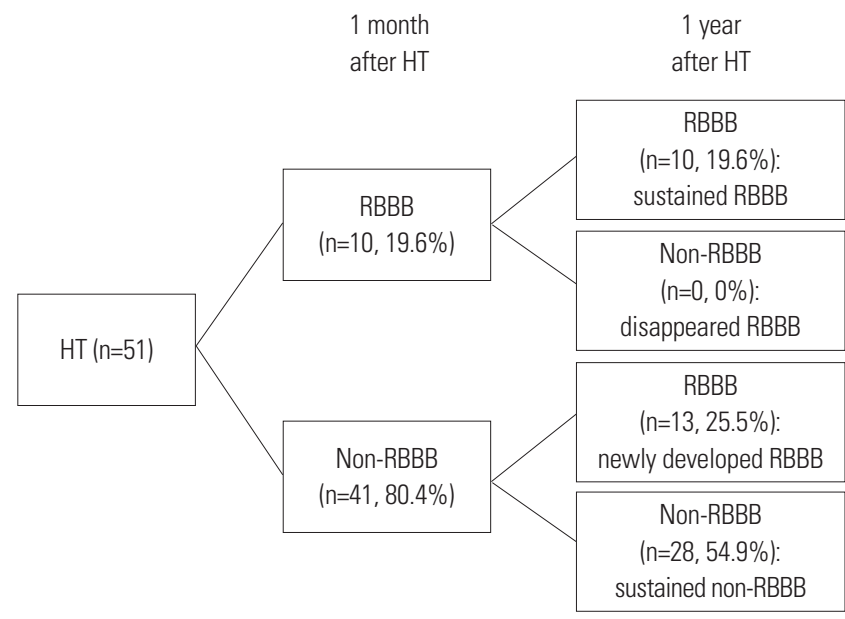

Fig. 1. Flow diagram of each group according to the development of RBBB after HT. HT, heart transplantation; RBBB, right bundle branch block. and at the outpatient clinic during follow-up. The RBBB was defined as an RSR' pattern in V1 with an R' amplitude of at least $0.2 \mathrm{mV}$ with a QRS duration $\geq 100 \mathrm{~ms}$ on ECG. ${ }^{6} \mathrm{PR}$ interval was defined as the interval from the onset of the $\mathrm{P}$ wave to the end of the PR segment, and was measured from the limb lead in which the interval was longest. ${ }^{10}$ The diagnosis of acute graft rejection was made by endomyocardial biopsy according to the International Society for Heart and Lung Transplantation (ISHLT) 2004 grading system: Grade 0, no rejection; Grade 1R, mild; Grade 2R, moderate; or Grade 3R, severe. ${ }^{11}$

\section{Echocardiography and right-side cardiac catheterization}

Two-dimensional echocardiography was performed to measure the dimensions and volume of the left ventricle (LV) and left atrium (LA), and LV ejection fraction was measured by the Simpson method as recommended. ${ }^{12}$ Right-side cardiac catheterization was performed to acquire hemodynamic data simultaneously during endomyocardial biopsy, and the following variables were measured: systolic, diastolic, and mean pressure of the pulmonary artery (PA) and right ventricle (RV) and pulmonary capillary wedge pressure.

\section{Statistical analysis}

Continuous variables including hemodynamic and echocardiographic parameters are expressed as mean $\pm \mathrm{SD}$, and they were compared using Student's t test. Discrete variables are expressed as percentages and were compared using the chi-square test and Fisher exact test for small groups. All statistical analyses were conducted using SPSS statistical software (ver. 21.0; IBM Corp., Armonk, NY, USA). A two-sided $p$ value of $<0.05$ was considered as statistically significant.

\section{RESULTS}

\section{Patient clinical characteristics}

The mean age of all donors was $33.6 \pm 11.0$ years, and $64.7 \%$ were male. The mean age of all recipients was $41.7 \pm 15.1$ years, and $58.8 \%$ were male. Table 1 shows the patient clinical characteristics of each group. Total ischemic time and immunosuppressive drug regimens during follow up were not different among each group. The prevalence of RBBB in our study was $19.6 \%$ (n= $10)$ at 1 month and $45.1 \%(n=23)$ at 1 year after HT. There were 10 patients (19.6\%) with sustained RBBB, 0 patients $(0 \%)$ with disappeared RBBB, 13 patients (25.5\%) with newly developed RBBB, and 28 patients (54.9\%) with sustained non-RBBB.

\section{Echocardiographic and invasive hemodynamic parameters according to the development of RBBB}

Details on RBBB development on ECG, right-side cardiac catheterization, echocardiography, and endomyocardial biopsy in each group are listed in Table 2. The newly developed RBBB 
Jin-Ho Kim, et al.

Table 1. Patient Clinical Characteristics

\begin{tabular}{|c|c|c|c|c|}
\hline & $\begin{array}{c}\text { Sustained RBBB } \\
(\mathrm{n}=10,19.6 \%)\end{array}$ & $\begin{array}{l}\text { Newly developed } \\
\text { RBBB }(n=13,25.5 \%)\end{array}$ & $\begin{array}{l}\text { Sustained non-RBBB } \\
\quad(n=28,54.9 \%)\end{array}$ & $p$ value \\
\hline \multicolumn{5}{|l|}{ Donor data } \\
\hline Male sex & $9(90)$ & $7(53.8)$ & $17(60.7)$ & 0.147 \\
\hline Age (yr) & $30.4 \pm 9.5$ & $38.0 \pm 10.5$ & $32.7 \pm 11.5$ & 0.223 \\
\hline Body surface area $\left(\mathrm{m}^{2}\right)$ & $1.76 \pm 0.17$ & $1.74 \pm 0.17$ & $1.76 \pm 0.17$ & 0.690 \\
\hline Preoperative LVEF (\%) & $64.9 \pm 6.9$ & $59.7 \pm 6.1$ & $62.7 \pm 6.7$ & 0.180 \\
\hline Cause of death on donor & & & & 0.117 \\
\hline Hypoxic brain damage & $2(20.0)$ & $3(23.1)$ & $9(32.1)$ & \\
\hline Non-traumatic brain hemorrhage & $6(60.0)$ & $2(15.4)$ & $4(14.3)$ & \\
\hline Traumatic brain hemorrhage & $2(20.0)$ & $7(53.8)$ & $14(50.0)$ & \\
\hline Others & $0(0.0)$ & $1(7.7)$ & $1(3.6)$ & \\
\hline \multicolumn{5}{|l|}{ Recipient data } \\
\hline Male sex & $5(50.0)$ & $9(69.2)$ & $16(57.1)$ & 0.596 \\
\hline Age (yr) & $38.3 \pm 16.0$ & $44.9 \pm 14.5$ & $41.5 \pm 15.1$ & 0.583 \\
\hline Body surface area $\left(\mathrm{m}^{2}\right)$ & $1.65 \pm 0.25$ & $1.71 \pm 0.23$ & $1.68 \pm 0.19$ & 0.470 \\
\hline Preoperative LVEF (\%) & $23.9 \pm 9.9$ & $25.6 \pm 9.0$ & $22.2 \pm 14.0$ & 0.694 \\
\hline Total ischemic time (min) & $163.7 \pm 53.6$ & $159.0 \pm 36.9$ & $145.7 \pm 36.5$ & 0.401 \\
\hline Previous open-heart surgery history & $3(30.0)$ & $7(53.8)$ & $6(21.4)$ & 0.125 \\
\hline
\end{tabular}

LVEF, left ventricular ejection fraction; RBBB, right bundle branch block.

Values are presented as $n(\%)$ or mean \pm SD.

group had significantly longer PR interval at 1 year than the sustained RBBB group and sustained non-RBBB group $(164.8 \pm 13.7$ ms vs. $148.6 \pm 25.7 \mathrm{~ms}$ vs. $149.2 \pm 18.3 \mathrm{~ms}$, respectively, $p=0.044$ ). However, no invasive hemodynamic parameters measured by right-side cardiac catheterization were different between each group at 1 month and 1 year following HT. LA volume index was significantly higher in the newly developed RBBB group than the sustained RBBB and sustained non-RBBB groups $\left(60.6 \pm 25.9 \mathrm{~mL} / \mathrm{m}^{2}\right.$ vs. $36.0 \pm 11.0 \mathrm{~mL} / \mathrm{m}^{2}$ vs. $38.4 \pm 18.1 \mathrm{~mL} / \mathrm{m}^{2}$, respectively, $p=0.003$ ). $\mathrm{LV}$ ejection fraction and parameters related with LV diastolic function were not significantly different among the groups.

\section{Graft rejection after HT}

As shown in Table 3, we identified 4 cases (30.8\%) of new onset graft rejection (from grade 0 to grade $\geq 1 \mathrm{R}$ ) in the newly developed RBBB group, which was higher than that in the other groups ( 1 case, $10.0 \%$ in sustained RBBB vs. 6 cases, $21.4 \%$ in sustained non-RBBB group). Interestingly, 7 patients $(70.0 \%)$ in the sustained RBBB group experienced resolved histologic finding (from grade $\geq 1 \mathrm{R}$ to grade 0 ) over time, compared to other groups (7.7\% in newly developed RBBB vs. $25.0 \%$ in sustained non-RBBB group). Among three patients with graft rejection Grade 2R at 1 year, two patients were in the newly developed RBBB group; one patient was in the sustained non-RBBB group.

In our HT patients, the PR interval (142.6 \pm 15.6 ms vs. 152.7 \pm $19.9 \mathrm{~ms}, p=0.002$ ) and QRS duration ( $87.2 \pm 13.3 \mathrm{~ms}$ vs. $97.0 \pm 14.8$ $\mathrm{ms}, p<0.001$ ) increased significantly from 1 month to 1 year after HT. Furthermore, 9 patients $(81.8 \%)$ with new onset graft rejection (from grade 0 to grade $\geq 1 \mathrm{R}$ ) had a significantly longer PR ( $\geq 153 \mathrm{~ms}$ ) interval at 1 year than other groups (58.3\% in no change of GR vs. $38.5 \%$ in no evidence of GR vs. $26.7 \%$ in resolving of GR) (Table 4).

\section{DISCUSSION}

The principal findings of this study are that 1) newly developed RBBB was associated with higher new onset graft rejection, higher LA volume index, and longer PR interval at 1 year and that 2) individuals with sustained RBBB experienced a significantly higher incidence of resolved graft rejection at 1 year, compared with newly developed RBBB and sustained nonRBBB.

The occurrence of conduction abnormalities after HT is well known, and the most common of these is RBBB. The prevalences of RBBB in the present study population were $15.9 \%$ at 1 month and $36.5 \%$ at 1 year, similar to previous studies ( $12 \%$ to $79 \%$ ). Most previous studies reported that the development of RBBB and QRS width increased over the time after HT. ${ }^{4,13-15}$ Meanwhile, several studies indicated that the occurrence of conduction disorders after HT was related with longer ischemic time, extracorporeal circulation time, surgery-related myocardial damage, and graft rejection., ${ }^{3,16}$ Among conduction disorders, the occurrence of RBBB immediately within 1 month after HT could pose different clinical implications from those with the occurrence of RBBB later. Since the right bundle branch has a unique anatomical structure, it might be susceptible to damage during HT: much of its septal course is near the en- 
Table 2. Electrocardiographic, Echocardiographic, and Invasive Hemodynamic Parameters and Endomyocardial Biopsy Results in Each Group

\begin{tabular}{|c|c|c|c|c|c|c|}
\hline & \multicolumn{2}{|c|}{ Sustained RBBB ( $n=10)$} & \multicolumn{2}{|c|}{ Newly developed RBBB ( $n=13$ ) } & \multicolumn{2}{|c|}{ Sustained non-RBBB ( $\mathrm{n}=28$ ) } \\
\hline & 1 month & 1 year & 1 month & 1 year & 1 month & 1 year \\
\hline \multicolumn{7}{|l|}{ Electrocardiogram } \\
\hline QRS width (ms) & $109.6 \pm 11.1^{*}$ & $112.6 \pm 8.8$ & $87.0 \pm 10.4$ & $110.6 \pm 12.1$ & $82.0 \pm 8.7$ & $90.1 \pm 10.1^{\|}$ \\
\hline PR interval (ms) & $133.4 \pm 13.0$ & $148.6 \pm 25.7$ & $143.8 \pm 14.6$ & $164.8 \pm 13.7^{\S}$ & $145.4 \pm 16.5$ & $149.2 \pm 18.3$ \\
\hline OTc (ms) & $456.8 \pm 27.0$ & $466.5 \pm 22.2$ & $446.0 \pm 25.8$ & $441.9 \pm 23.0$ & $446.0 \pm 25.8$ & $441.9 \pm 23.0$ \\
\hline \multicolumn{7}{|l|}{ Right heart catheterization } \\
\hline RVSP (mm Hg) & $30.2 \pm 7.9$ & $28.3 \pm 7.4$ & $31.1 \pm 6.6$ & $26.3 \pm 6.8$ & $30.3 \pm 6.6$ & $28.4 \pm 7.8$ \\
\hline $\operatorname{RVDP}(\mathrm{mm} \mathrm{Hg})$ & $4.5 \pm 7.2$ & $1.3 \pm 4.0$ & $2.6 \pm 2.4$ & $0.1 \pm 3.5$ & $3.2 \pm 3.3$ & $1.6 \pm 2.7$ \\
\hline $\mathrm{RVMP}(\mathrm{mm} \mathrm{Hg})$ & $16.0 \pm 7.2$ & $12.4 \pm 2.7$ & $14.8 \pm 4.7$ & $11.0 \pm 5.1$ & $15.0 \pm 3.8$ & $14.4 \pm 5.1$ \\
\hline PASP (mm Hg) & $27.6 \pm 8.5$ & $26.5 \pm 5.7$ & $29.1 \pm 6.2$ & $24.8 \pm 7.5$ & $29.0 \pm 7.7$ & $27.4 \pm 6.5$ \\
\hline PADP (mm Hg) & $10.7 \pm 2.9$ & $11.3 \pm 2.8$ & $13.4 \pm 4.3$ & $9.9 \pm 4.4$ & $12.3 \pm 4.6$ & $12.8 \pm 4.7$ \\
\hline PAMP (mm Hg) & $18.3 \pm 3.2$ & $18.2 \pm 3.9$ & $18.0 \pm 5.7$ & $17.0 \pm 5.7$ & $19.7 \pm 5.8$ & $19.7 \pm 5.4$ \\
\hline PCWP (mm Hg) & $8.7 \pm 4.3$ & $9.6 \pm 2.8$ & $12.8 \pm 4.5$ & $9.2 \pm 3.8$ & $10.7 \pm 3.3$ & $12.1 \pm 6.1$ \\
\hline \multicolumn{7}{|l|}{ Echocardiographic data } \\
\hline $\operatorname{LVEF}(\%)$ & $68.2 \pm 6.3$ & $65.2 \pm 6.8$ & $66.0 \pm 7.2$ & $68.2 \pm 4.3$ & $67.5 \pm 5.5$ & $65.0 \pm 7.9$ \\
\hline LVMI (g/m²) & $94.1 \pm 21.5$ & $84.5 \pm 13.1$ & $106.9 \pm 36.7$ & $93.9 \pm 28.6$ & $94.2 \pm 24.9$ & $81.7 \pm 17.3$ \\
\hline LAVI $\left(\mathrm{mL} / \mathrm{m}^{2}\right)$ & $38.6 \pm 12.8$ & $36.0 \pm 11.0$ & $63.2 \pm 24.1^{\dagger}$ & $60.6 \pm 25.9^{\ddagger}$ & $39.2 \pm 13.9$ & $38.4 \pm 18.1$ \\
\hline E velocity (m/s) & $0.71 \pm 0.12$ & $0.70 \pm 0.21$ & $0.83 \pm 0.27$ & $0.79 \pm 0.23$ & $0.73 \pm 0.21$ & $0.75 \pm 0.17$ \\
\hline$E^{\prime}$ velocity $(\mathrm{cm} / \mathrm{s})$ & $6.3 \pm 2.4$ & $8.0 \pm 2.8$ & $5.7 \pm 2.2$ & $7.1 \pm 2.7$ & $6.2 \pm 1.8$ & $7.7 \pm 2.0$ \\
\hline $\mathrm{E} / \mathrm{E}^{\prime}$ & $12.4 \pm 3.7$ & $9.3 \pm 3.1$ & $15.2 \pm 5.8$ & $11.8 \pm 4.1$ & $12.7 \pm 4.5$ & $10.2 \pm 2.8$ \\
\hline $\operatorname{RVSP}(\mathrm{mm} \mathrm{Hg})$ & $33.8 \pm 9.1$ & $29.2 \pm 8.4$ & $35.1 \pm 11.6$ & $26.0 \pm 8.7$ & $34.6 \pm 9.3$ & $29.5 \pm 5.1$ \\
\hline TAPSE (mm) & $14.0 \pm 3.9$ & $16.8 \pm 0.6$ & $15.6 \pm 3.4$ & $16.0 \pm 2.6$ & $15.7 \pm 2.9$ & $15.9 \pm 2.2$ \\
\hline TV TDI S' (cm/s) & $9.1 \pm 1.8$ & $10.2 \pm 2.0$ & $8.6 \pm 3.2$ & $10.0 \pm 2.2$ & $8.6 \pm 2.4$ & $9.3 \pm 2.6$ \\
\hline \multicolumn{7}{|l|}{ Histology } \\
\hline Graft rejection ( $\geq 1)$ & $7(70.0)$ & $1(10.0)$ & $6(46.2)$ & $9(69.2)$ & $14(50.0)$ & $13(46.4)$ \\
\hline \multicolumn{7}{|l|}{ Immunosuppressant level } \\
\hline Tacrolimus level (ng/mL) & $10.9 \pm 5.8$ & $9.5 \pm 3.8$ & $8.9 \pm 2.1$ & $7.9 \pm 2.2$ & $9.5 \pm 3.5$ & $9.8 \pm 3.1$ \\
\hline
\end{tabular}

LAVI, left atrial volume index; LVMI, left ventricular mass index; PCWP, pulmonary capillary wedge pressure; RBBB, right bundle branch block; RVSP, right ventricular systolic pressure; RVDP, right ventricular diastolic pressure; RVMP, right ventricular mean pressure; PASP, pulmonary artery systolic pressure; PADP, pulmonary artery diastolic pressure; PAMP, pulmonary artery mean pressure; PCWP, pulmonary capillary wedge pressure; TAPSE, tricuspid annular plane systolic excursion; TV TDI S', tricuspid valve tissue Doppler imaging S'.

Values are expressed as mean $\pm S D$ or number (percentage).

$p<0.05$, by ANOVA with post hoc test using Bonferroni technique (* ${ }^{*}$ sustained RBBB group vs. newly developed RBBB group and sustained non-RBBB group at 1 month following heart transplantation; ${ }^{\dagger}$ newly developed RBBB group vs. sustained RBBB group and sustained non-RBBB group at 1 month following heart transplantation; " newly developed RBBB group vs. sustained RBBB group and sustained non-RBBB group at 1 year following heart transplantation; ${ }^{\S}$ newly developed RBBB group vs. sustained non-RBBB group at 1 year following heart transplantation; "Isustained non-RBBB group vs. sustained RBBB group and newly developed RBBB group at 1 year following heart transplantation).

docardium and consists of narrow cylindrical tubes. ${ }^{17}$ Also, biatrial anastomosis and clockwise rotation of a transplanted heart, as a result of the surgical technique, could be other causative factors for RBBB. ${ }^{1,15,18,19}$ Considering these factors, our study indicated that the occurrence of RBBB early within 1 month following HT was less likely to be related with early graft rejection.

Our study showed that the newly developed RBBB after 1 month later following HT could be related with graft rejection. As in our findings, Osa, et al. ${ }^{6}$ showed that progressive RBBB, during which QRS width increases more than $0.5 \mathrm{~mm}$ during follow-up, was associated with a poorer long-term prognosis than non-progressive RBBB and related to a greater number of treatable rejections. Also, patients with gradually worsening conduction abnormalities exhibited significant differences in the number of rejections to treatment, suggesting a correlation between the progression of conduction abnormalities and poor clinical outcomes, as described in the study of Leonelli, et al. ${ }^{3,20}$ On the other hand, the sustained RBBB group in our study had a relatively low rejection rate and a higher resolution of histologic findings during follow up, compared with the newly developed RBBB group. The absence of changes in ECG during follow up might be more histologically stable, although further research is needed to evaluate and clarify this.

Mild graft rejection, such as Grade $1 \mathrm{R}$ on the revised ISHLT grading system, was previously found to be the strongest predictor for severe graft rejection of more than Grade 2R, suggesting short-term follow up seems to be warranted. ${ }^{21}$ Furthermore, 
Table 3. Graft Rejection after HT in Each Group

\begin{tabular}{|c|c|c|c|c|c|}
\hline & $\begin{array}{c}\text { No change of GR } \\
(n=12,23.5 \%)\end{array}$ & $\begin{array}{c}\text { No evidence of GR } \\
(n=13,25.5 \%)\end{array}$ & $\begin{array}{c}\text { New onset of GR } \\
(n=11,21.6 \%)\end{array}$ & $\begin{array}{c}\text { Resolving of GR } \\
(n=15,29.4 \%)\end{array}$ & $p$ value \\
\hline Sustained RBBB $(\mathrm{n}=10)$ & $0(0.0)$ & $2(20.0)$ & $1(10.0)$ & $7(70.0)$ & \multirow{3}{*}{0.042} \\
\hline Newly developed RBBB ( $n=13$ ) & $5(38.5)$ & $3(23.1)$ & $4(30.8)$ & $1(7.7)$ & \\
\hline Sustained non-RBBB (n=28) & $7(25.0)$ & $8(28.6)$ & $6(21.4)$ & $7(25.0)$ & \\
\hline
\end{tabular}

$\mathrm{HT}$, heart transplantation; $\mathrm{GR}$, graft rejection; RBBB, right bundle branch block.

Values are presented as $n(\%)$. $p$ value by chi-square test.

Table 4. Graft Rejection according to PR Interval at 1 Year after HT

\begin{tabular}{lccccc}
\hline & \multicolumn{5}{c}{ The change of graft rejection from 1 month to 1 year following HT } \\
\cline { 2 - 5 } & No change in GR & No evidence of GR & New onset of GR & Resolving of GR & $\boldsymbol{p}$ value \\
\hline PR interval $\left(<153 \mathrm{~ms}^{*}\right)$ & $5(41.7)$ & $8(61.5)$ & $2(18.2)$ & $9(81.8)$ & $11(73.3)$ \\
PR interval $(\geq 153 \mathrm{~ms})$ & $7(58.3)$ & $5(38.5)$ & $4(26.7)$ & 0.033 \\
\hline
\end{tabular}

$\mathrm{HT}$, heart transplantation; GR, graft rejection; RBBB, right bundle branch block.

Values are presented as $n(\%)$. $p$ value by chi-square test.

*153 ms: median value of PR interval in overall study population at 1 year following HT.

mild graft rejection at the conduction system, including the atrioventricular (AV) node and right bundle branch, could be associated with the development of RBBB, PR interval prolongation, and AV block. ${ }^{8}$ Prolonged PR interval, another type of conduction abnormality, was linked to conduction system fibrosis, and graft rejection. ${ }^{10,22}$ Some studies reported AV block was associated with a higher risk of graft rejection, which resulted from lymphocyte infiltration, inflammation, myocyte necrosis or fibrosis at the conduction system. ${ }^{23-26}$ Furthermore, Calzolari, et al. ${ }^{8}$ reported that appearance of first-degree AV block in a HT recipient may be suggestive of involvement of the conduction system with impending third-degree block. Also, Chan, et al. ${ }^{9}$ reported early signs of first degree AV block after HT may portend aggravated conduction abnormalities with occurrence of subclinical, isolated rejection of the conduction system. In our study, at 1 year after HT, newly developed RBBB posed a significantly longer PR interval, and PR interval in patients who had new onset graft rejection at 1 year was significantly longer than those in patients without new onset graft rejection ( $161.5 \pm 12.2 \mathrm{~ms}$ vs. $150.8 \pm 20.9 \mathrm{~ms}, p=0.041$ ) (detailed in Supplementary Table 1, only online). Accordingly, we suggest that newly developed RBBB with a relatively longer PR interval at 1 year following HT might be strongly associated with graft rejection involving conduction system.

Our study had several limitations. First, this study was a retrospective single-center study, even though the data were collected in a prospective manner. Second, our study population was too small, and the follow-up period was too short, only up to 1 year. Third, endomyocardial biopsies were just performed two times at 1 month and at 1 year following HT. It remains still unknown what kind of histologic changes have occurred in relation to ECG between 1 month and 1 year after HT. However, a recent study showed that morbidity and mortality of lower frequency routine surveillance EMB were comparable with data from the ISHLT registry. ${ }^{27}$ Fourth, we did not analyze these new parameters for graft rejection in all the HT re- cipients. Considering the prognostic importance of antibody mediated rejection and donor specific antibody in HT recipients, further study of the relationship between RBBB and these parameters is warranted. Fifth, we did not measure rejectionrelated biomarkers, such as natriuretic peptide, CK-MB, and troponin, serially in all HT recipients. Sixth, we could not obtain and analyze the ECG of every donor heart in this study. Finally, clinical outcomes, such as sudden cardiac death or occurrence of heart failure, related primarily to conduction disturbance or acute graft rejection were not analyzed. Further larger longterm follow-up study is warranted to confirm our hypothesisgenerating findings. In conclusion, close monitoring for newly developed RBBB and a relatively longer PR interval at 1 year following HT may help to identify patients at high risk for graft rejection.

\section{AUTHOR CONTRIBUTIONS}

Conceptualization: Jaewon Oh, Seok-Min Kang. Data curation: Min Ji Kim. Formal analysis: In-Cheol Kim. Methodology: Jin-Ho Kim. Supervision: Hui-Nam Pak. Validation: Jae-Sun Uhm. Visualization: JinHo Kim. Writing_original draft: Jin-Ho Kim. Writing_review \& editing: Jin-Ho Kim, Jaewon Oh, Seok-Min Kang.

\section{ORCID iDs}

Jin-Ho Kim Jaewon Oh Min Ji Kim In-Cheol Kim Jae-Sun Uhm Hui-Nam Pak Seok-Min Kang

https://orcid.org/0000-0003-3585-7859 https://orcid.org/0000-0002-4585-1488 https://orcid.org/0000-0002-5332-9673 https://orcid.org/0000-0002-5751-2328 https://orcid.org/0000-0002-1611-8172 https://orcid.org/0000-0002-3256-3620 https://orcid.org/0000-0001-9856-9227

\section{REFERENCES}

1. Sandhu JS, Curtiss EI, Follansbee WP, Zerbe TR, Kormos RL. The scalar electrocardiogram of the orthotopic heart transplant recip- 
ient. Am Heart J 1990;119:917-23.

2. Gao SZ, Hunt SA, Wiederhold V, Schroeder JS. Characteristics of serial electrocardiograms in heart transplant recipients. Am Heart J 1991;122(3 Pt 1):771-4.

3. Leonelli FM, Pacifico A, Young JB. Frequency and significance of conduction defects early after orthotopic heart transplantation. Am J Cardiol 1994;73:175-9.

4. Jessen ME, Olivari MT, Wait MA, Meyer DM, Yancey CW Jr, Ring WS. Frequency and significance of right bundle branch block after cardiac transplantation. Am J Cardiol 1994;73:1009-11.

5. Golshayan D, Seydoux C, Berguer DG, Stumpe F, Hurni M, Ruchat P, et al. Incidence and prognostic value of electrocardiographic abnormalities after heart transplantation. Clin Cardiol 1998;21:680-4.

6. Osa A, Almenar L, Arnau MA, Martínez-Dolz L, Rueda J, Morillas $\mathrm{P}$, et al. Is the prognosis poorer in heart transplanted patients who develop a right bundle branch block? J Heart Lung Transplant 2000; 19:207-14.

7. Foerster A. The conduction system in human cardiac allografts. A histological and immunopathological study. Pathol Res Pract 1992; 188:783-90.

8. Calzolari V, Angelini A, Basso C, Livi U, Rossi L, Thiene G. Histologic findings in the conduction system after cardiac transplantation and correlation with electrocardiographic findings. Am J Cardiol 1999;84:756-9.

9. Chan JB, Levi DS, Lai CK, Alejos JC, Fishbein MC. Cellular rejection of the conduction system after orthotopic heart transplantation for congenital atrioventricular block. J Heart Lung Transplant 2006;25:1371-5.

10. Aro AL, Anttonen O, Kerola T, Junttila MJ, Tikkanen JT, Rissanen HA, et al. Prognostic significance of prolonged PR interval in the general population. Eur Heart J 2014;35:123-9.

11. Stewart S, Winters GL, Fishbein MC, Tazelaar HD, Kobashigawa J, Abrams J, et al. Revision of the 1990 working formulation for the standardization of nomenclature in the diagnosis of heart rejection. J Heart Lung Transplant 2005;24:1710-20.

12. Lang RM, Bierig M, Devereux RB, Flachskampf FA, Foster E, Pellikka PA, et al. Recommendations for chamber quantification: a report from the American Society of Echocardiography's Guidelines and Standards Committee and the Chamber Quantification Writing Group, developed in conjunction with the European Association of Echocardiography, a branch of the European Society of Cardiology. J Am Soc Echocardiogr 2005;18:1440-63.

13. Marcus GM, Hoang KL, Hunt SA, Chun SH, Lee BK. Prevalence, patterns of development, and prognosis of right bundle branch block in heart transplant recipients. Am J Cardiol 2006;98:1288-90.

14. Villa AE, de Marchena EJ, Myerburg RJ, Castellanos A. Comparisons of paired orthotopic cardiac transplant donor and recipient electrocardiograms. Am Heart J 1994;127:70-4

15. Ferretto S, Tafciu E, Giuliani I, Feltrin G, Bottio T, Gambino A, et al. Interventricular conduction disorders after orthotopic heart transplantation: risk factors and clinical relevance. Ann Noninvasive Electrocardiol 2017;22:e12402.

16. Martí S, Almenar L, Luis Vicente J, Torregrosa S, Roldán I, Moreno MT, et al. [Which variables predict the appearance of right bundlebranch block and its course after heart transplantation?]. Rev Esp Cardiol 1996;49:328-33.

17. Thalen HJTh, Meere CC. Fundamentals of cardiac pacing. 1st ed. The Hague: Springer; 1979. p.44.

18. Butman SM, Phibbs B, Wild J, Copeland JG. One heart, two bodies: insight from the transplanted heart and its new electrocardiogram. Am J Cardiol 1990;66:632-5.

19. Alderman EL, Wexler L. Angiographic implications of cardiac transplantation. Am J Cardiol 1989;64:16E-21E.

20. Leonelli FM, Dunn JK, Young JB, Pacifico A. Natural history, determinants, and clinical relevance of conduction abnormalities following orthotopic heart transplantation. Am J Cardiol 1996;77:47-51.

21. Brunner-La Rocca HP, Sütsch G, Schneider J, Follath F, Kiowski W. Natural course of moderate cardiac allograft rejection (International Society for Heart Transplantation grade 2) early and late after transplantation. Circulation 1996;94:1334-8.

22. Kwok CS, Rashid M, Beynon R, Barker D, Patwala A, Morley-Davies A, et al. Prolonged PR interval, first-degree heart block and adverse cardiovascular outcomes: a systematic review and metaanalysis. Heart 2016;102:672-80.

23. Payne ME, Murray KD, Watson KM, Galbraith TA, Horwanitz EP, Starling RC, et al. Permanent pacing in heart transplant recipients: underlying causes and long-term results. J Heart Lung Transplant 1991;10(5 Pt 1):738-42.

24. Collins KK, Thiagarajan RR, Chin C, Dubin AM, Van Hare GF, Robbins RC, et al. Atrial tachyarrhythmias and permanent pacing after pediatric heart transplantation. J Heart Lung Transplant 2003;22: 1126-33.

25. Cannon BC, Denfield SW, Friedman RA, Fenrich AL, Dreyer WJ, Towbin JA, et al. Late pacemaker requirement after pediatric orthotopic heart transplantation may predict the presence of transplant coronary artery disease. J Heart Lung Transplant 2004;23:67-71.

26. Grutter G, Alfieri S, Calcagni G, Castelluzzo MA, Silvetti MS, Parisi F, et al. Paroxysmal atrioventricular block after heart transplantation in children: an early sign of rejection? Pediatr Transplant 2016;20: 1164-7.

27. Weckbach LT, Maurer U, Schramm R, Huber BC, Lackermair K, Weiss $\mathrm{M}$, et al. Lower frequency routine surveillance endomyocardial biopsies after heart transplantation. PLoS One 2017;12: e0182880. 